\title{
Individualized metacognitive therapy for delusions: A randomized controlled rater-blind study
}

\author{
Christina Andreou, MD, PhD ${ }^{\mathrm{a}, \mathrm{b}}$; Charlotte Wittekind, PhD ${ }^{\mathrm{b}}$; Martina Fieker, MSc ${ }^{\mathrm{b}}$; Ulrike \\ Heitz, MSca; Ruth Veckenstedt, PhD ${ }^{\text {; }}$ Francesca Bohn, PhD ${ }^{\text {; }}$ Steffen Moritz, PhD \\ a Center for Gender Research and Early Detection, University Psychiatric Clinics Basel, \\ Switzerland \\ b Department of Psychiatry and Psychotherapy, University Medical Center Hamburg-Eppendorf, \\ Germany
}

Corresponding author: Christina Andreou, Center for Gender Research and Early Detection, University Psychiatric Clinics Basel, Kornhausgasse 7, 4055 Basel, Switzerland. Tel +41 61325 8165, Fax +41 61325 8160, email: Christina.Andreou@upkbs.ch 


\section{Abstract}

Background \& Objectives: Theory-driven interventions targeting specific factors that contribute to delusions are receiving increased interest. The present study aimed to assess the efficacy of individualized metacognitive therapy (MCT+), a short manualized intervention that addresses delusion-associated cognitive biases.

Methods: 92 patients with current or past delusions were randomized to receive 12 twiceweekly sessions of either MCT+ or a control intervention within a randomized controlled raterblind design. Psychopathology and cognitive biases were assessed at baseline, 6 weeks and 6 months. ANCOVAs adjusted for baseline scores were used to assess differences between groups regarding outcome variables. Both per-protocol and intention-to-treat analyses were conducted.

Results: At 6 weeks, there was a significant difference in favor of MCT+ regarding decrease in delusion severity and improvement of self-reflectiveness (medium effect size), and a trend-wise difference regarding probability threshold to decision. These effects increased, when only patients attending a minimum of 4 therapy sessions were considered. Control group patients subsequently showed further improvement while patients in the MCT+ group remained stable, such that there were no differences between groups at the 6-month follow-up.

Limitations: Lower attendance rates in the control group possibly leading to unequal therapeutic effort; lower baseline delusion severity in the MCT+ group.

Conclusions: The result pattern suggests that MCT+ led to an earlier improvement in delusions and cognitive biases compared to the control intervention. The absence of a long-term effect might reflect floor effects in the MCT+ group, but may also indicate the need for further measures to promote sustainability of MCT+ effects.

Keywords: schizophrenia, metacognition, cognitive biases, jumping-to-conclusions, psychotherapy, cognitive-behavioral therapy. 


\section{Introduction}

Delusions are one of the most common and recognizable symptoms of psychotic disorders. Up until the late $20^{\text {th }}$ century, delusional beliefs were viewed as "non-understandable" (Jaspers, 1913), and biological conceptualizations predominated treatment approaches (Mander and Kingdon, 2015). However, a new picture has gradually emerged. Behavioral, cognitive and social studies but also social influences such as the consumer movement led to an increased awareness of cognitive and psychological factors in the emergence of delusions (Mander and Kingdon, 2015; Mueser et al., 2013). The concurrent growing realization of the limitations of antipsychotic medication, especially with respect to functional recovery (Jaaskelainen et al., 2013; Leucht et al., 2009) and adherence issues (Lieberman et al., 2005) have boosted interest in psychological interventions for the treatment of delusions.

Cognitive-behavioral therapy (CBT) has had a leading role in this field. Having provided a wide empirical basis supporting its efficacy in treating delusions (Hutton and Taylor, 2014; Turner et al., 2014; Wykes et al., 2008), CBT was one of the first psychological interventions to be included in treatment guidelines for psychosis. However, there is still an ongoing debate about its efficacy,(McKenna and Kingdon, 2014) especially when it comes to disentangling 'true' efficacy from unspecific therapy effects (Jauhar et al., 2014; Lynch et al., 2010; Mehl et al., 2015). In an effort to maximize efficacy, recent research has focused on targeted therapies that deal with individual factors thought to contribute to psychotic symptoms, such as worry (Freeman et al., 2015) or reasoning biases (Garety et al., 2015; Moritz et al., 2014a; Waller et al., 2011). It has been suggested that such theory-driven interventions may lead to improved outcomes compared to standard CBT (Mehl et al., 2015).

One of these refined approaches is metacognitive training (MCT), a manualized group intervention (Moritz et al., 2013b). MCT builds upon evidence associating delusional beliefs with specific thinking styles that lead to distorted appraisals of events (Garety and Freeman, 2013). Well-established examples include jumping-to-conclusions, overconfidence in false judgments, and belief inflexibility/incorrigibility. Importantly, these thinking styles, termed 'cognitive biases', are not symptom-specific, but rather an extension of normal thinking styles, appearing also in neutral (i.e. delusion-unrelated) contexts. MCT adopts a hands-on approach, aiming to raise patients' awareness for such cognitive biases. The ultimate goal is to 'plant the seeds of doubt' through entertaining and collaborative exercises that use predominantly non-delusional scenarios.

Several randomized controlled studies (Moritz et al., 2014a) as well as a recent meta-analysis (Eichner and Berna, 2016) have shown promising results regarding the short- and long-term efficacy of group MCT on delusions and/or positive psychotic symptoms in general (although 
there have also been negative results (van Oosterhout et al., 2014; van Oosterhout et al., 2016)). This effect appears to be complementary to that of antipsychotic medication, since all the above results were obtained using MCT as adjunctive treatment to patients already receiving antipsychotics. However, the group intervention format may not be suited for some patients, including those with high level of suspiciousness (van Oosterhout et al., 2014), or patients with negative and/or disorganized symptoms that may require more intensive and structured work (Moritz et al., 2005). On the other hand, it has been suggested that the effects of metacognitive interventions on reasoning and delusions might be promoted with use of personalized material and individual therapy sessions (Garety et al., 2015; van Oosterhout et al., 2014).

Previous studies have shown that use of MCT material in an individual treatment format can have beneficial effects on cognitive biases and/or delusions after very few sessions (Balzan et al., 2014; Balzan and Galletly, 2015; Ross et al., 2011; So et al., 2015; Waller et al., 2011). In a randomized, controlled, rater-blind trial of group MCT combined with individualized sessions (Moritz et al., 2011), patients in the MCT arm showed significantly greater improvement in delusion severity and conviction, as well as in jumping-to-conclusions, relative to the active control group. Interestingly, effect sizes were quite large $(d>0.6)$ for delusions in that study despite the short duration of the intervention and follow-up (4 weeks). The authors concluded that the application of MCT material to individual delusional beliefs might provide additional benefits compared to the group MCT; however, the sample size was too small to draw conclusive inferences.

Based on these findings, our group developed a fully individualized version of MCT. Metacognitive therapy (MCT+)(Moritz et al., 2012b) is a manualized intervention that, similar to MCT, targets common reasoning biases encountered in patients with delusions. However, MCT addresses the 'metacognitive infrastructure' of delusions solely with use of neutral exercises. In contrast, individualized MCT + follows up on this initial step by applying the learned material (using techniques adopted from CBT) to challenge the content of individual delusional beliefs.

So far, there have been no randomized clinical studies on MCT+. Therefore, the present study aimed to assess the efficacy of this intervention in patients with delusions compared to an active control condition, consisting in a cognitive training intervention. We designed the study as a randomized controlled, rater-blinded trial, while at the same time including as many 'pragmatic' aspects as possible (such as broad inclusion criteria and flexibility in intervention delivery) to ensure generalizability of results and inform planning of larger, multicenter trials on MCT+. We hypothesized that MCT+ would lead to significantly greater decline in delusion severity and dysfunctional reasoning compared to the control condition. 


\section{Materials and methods}

The study was conducted at the Department of Psychiatry and Psychotherapy of the University Medical Center Hamburg-Eppendorf (Germany). Participants were 92 patients with nonaffective psychotic disorders and current or past delusions, recruited among in- and outpatients treated at the Psychosis Center of the Department from January 2013 through July 2015 and judged by their attending psychiatrist to qualify for study participation. Inclusion criteria were age 18 to 65 years, a DSM-IV diagnosis of a schizophrenia spectrum disorder confirmed with the Mini Neuropsychiatric Interview (Sheehan et al., 1998), and a present or prior delusional episode. Exclusion criteria were kept to a minimum in order to ensure generalizability of findings, and included a primary diagnosis of substance use disorder, $\mathrm{IQ}<70$, severe organic brain disorders, previous experience with group MCT or any of the experimental interventions, and any ongoing CBT-oriented psychotherapy. The trial was approved by the ethics committee of the German Psychology Association, and all patients gave their written informed consent before entering the study. A CONSORT diagram is provided in Figure 1.

Patients were randomized according to a computerized randomization plan [pseudorandom fixed procedure, analogous to a previous group MTC trial by our group (Moritz et al., 2014b; Moritz et al., 2013a)] to one of two interventions: MCT+ or CogPack® (Marker, 2003) (see below for details regarding the interventions). Treatment arm allocation was performed observerblind and communicated to patients by a person who was neither involved in the assessments nor in intervention delivery. All patients continued to receive their usual treatment throughout study participation. Importantly, as group MCT is a standard part of treatment in our department, patients from both groups were allowed to take part in MCT groups during study participation. However, this information was documented and considered in analyses.

Assessments were carried out at baseline, at 6 weeks (T1, corresponding to completion of 12 intervention sessions) and 6 months later (T2). All assessments were carried out by raters blind to treatment allocation. Rater training was performed according to the same procedure used in our recent group MCT study (Moritz et al., 2013a). In order to further enhance reliability, assessments for each individual patient were carried out by the same rater throughout the trial period.

\subsection{Outcomes}

Psychopathology was assessed with the Psychotic Symptom Rating Scales (PSYRATS) (Haddock et al., 1999) and the Positive and Negative Syndrome Scale (PANSS) (Kay et al., 1987). Both instruments have been widely used in intervention studies and have good psychometric properties (Drake et al., 2007; Peralta and Cuesta, 1994). The main outcome of interest was 
delusion severity at T1 as reflected in the delusion subscale total score of the PSYRATS. Secondary outcomes included PSYRATS delusion score at T2, PANSS P1 item (Delusions) at T1 and T2, and psychopathology according to the 5-factor model of the PANSS (Wallwork et al., 2012), at $\mathrm{T} 1$ and $\mathrm{T} 2$.

Further secondary outcomes of interest (assessed both at T1 and T2) included the following:

- The Fish Task (Moritz et al., 2012a), a computerized variant of the Beads Task, was used to assess jumping-to-conclusions, a prototypical cognitive bias. In the task, participants are presented with two lakes containing fish in opposite color ratios (80:20 orange:gray or viceversa). Ten fish are successively presented in a predetermined sequence to the participant. After each draw, the participant is required to estimate the probability that fish originated from lake $\mathrm{A}$, and to indicate whether they have made a decision regarding the origin of the fish. All fish drawn remain visible throughout the task in order to minimize working memory demands. Parallel versions were used across the testing sessions to reduce practice effects. The variables of interest were the number of draws to decision, as well as the probability threshold at decision (i.e., the minimum probability estimate, at which a decision was made in favor of the respective lake; a higher probability threshold indicates more cautious inference making).

- The World Health Organization Quality of Life - BREF (WHOQOL-BREF) (Murphy et al., 2000) was used as a measure of overall life satisfaction. This self-report scale assesses quality of life in four domains: physical, psychological, social and environment. Moreover, two global items assess overall quality of life and general health satisfaction.

- The Rosenberg Self-Esteem Scale (von Collani and Herzberg, 2003), a widely used 10-item self-report measure, was administered to assess self-esteem.

- The Beck Cognitive Insight Scale (BCIS) (Beck et al., 2004) measures the ability to distance oneself from one's own ideas and reflect upon their possible fallibility. The 15-item selfreport measure yields two scores reflecting self-reflectiveness and self-certainty. It has been suggested (Beck and Warman, 2004) and confirmed in patient studies (Riggs et al., 2012) that these cognitive insight indices are related to delusional thinking in particular among psychotic symptoms, as they reflect inflexible reasoning styles that support delusional beliefs.

\subsection{Interventions}

\subsubsection{Experimental intervention}

MCT+ is a manualized intervention that comprises 12 twice-weekly individual therapy sessions. Its main goal is to highlight the fallibility of cognition in general and encourage patients to reflect on their own thinking styles in relation to symptoms, but also to everyday life. MCT+ has a 
modular structure. Three introductory modules focus on history taking, introduction to the intervention rationale and development of a personal illness model. The major cognitive biases described above are each introduced in separate modules, and this knowledge is used in later modules to discuss broader topics such as social interaction, mood and stress coping. Other than the three introductory modules, presentation of individual modules was not fixed in the present study, but rather tailored to the individual needs and metacognitive abilities of the patient as judged by the therapist. Thus, it was possible to spend more time on a module, revisit some and skip other modules. Sessions lasted approximately 45-60 min. Most sessions included homework tasks according to the principles of CBT.

Therapy was delivered by psychologists with variable expertise (and mostly in various stages of their training in psychotherapy) in order to best reflect standard conditions in patient care. All therapists received group supervision by a certified psychotherapist.

\subsubsection{Control intervention}

In order to match the two patient groups on therapeutic effort, an active control condition was used. The latter consisted in $\operatorname{CogPack}^{\circledR}$ (Marker, 2003), a computerized cognitive training program that targets cognitive dysfunctions commonly encountered in patients with psychosis. Treatment was administered individually on personal computers and covered a wide range of neuropsychological exercises involving memory, reasoning, selective attention and psychomotor speed. Each session lasted approximately 45-60 min. Similarly to MCT+, patients could receive a maximum of 12 consecutive sessions.

\subsection{Statistical analyses}

Differences between groups in gender, age, premorbid IQ baseline symptom severity and antipsychotic dose, as well as mean antipsychotic medication dose over the whole study were assessed by means of t-tests.

Both intention-to-treat (ITT) and per protocol (PP) analyses were conducted. For PP analyses, participants were required to participate in post-treatment and follow-up assessments, respectively. ITT analysis considered data from all participants with available baseline data. Multiple imputation was adopted to estimate post-treatment and follow-up scores for noncompleters. Both types of analyses used ANCOVAs to assess differences regarding outcome variables between groups at $\mathrm{T} 1$ and $\mathrm{T} 2$. In each of these ANCOVAS, change score of the respective variable (e.g. PSYRATS delusions change score for the primary outcome) was the dependent variable. Independent variable was group allocation (MCT+ vs. CogPack $\left.{ }^{\circledR}\right)$. The baseline score of the outcome variable was included as a covariate in the model. The reported 
results include also gender and IQ as predictors, although conducting analyses without these variables led to no changes at all regarding the direction of differences and significance levels. Significant results are reported at $\mathrm{p}<0.05$ (two-sided), and statistical trends at $\mathrm{p}<0.1$. Effect sizes are expressed using $\eta^{2}$ partial, whereby .01 is equivalent to a small effect, .06 is equivalent to a medium effect and .14 is equivalent to a strong effect (Kinnear and Gray, 2009).

Sample size calculations performed with Gpower (Erdfelder et al., 1996) indicated that a total sample size of 90 would be sufficient to detect an effect in the medium range for the primary outcome $\left(\eta^{2}\right.$ partial $\left.=0.08\right)$, for $\mathrm{a}=0.05$ and $\beta=0.20$.

\section{Results}

\subsection{Sample characteristics}

The two groups did not significantly differ in gender, age, premorbid IQ (as assessed with a German vocabulary test (Schmidt and Metzler, 1992)) and years of education (Table 1). About one third of patients concurrently participated in the MCT group program (a standard part of treatment in our department, see Section 2), and there were no differences between the two treatment groups in this regard $\left(M C T+n=15 ; \operatorname{CogPack} \circledast n=20 ; \chi^{2}(1)=1.35, p=0.25\right)$. There were also no differences in antipsychotic medication dose either at baseline or in average over the whole study period (Table 1).

There were some baseline differences in symptoms between the two intervention groups, with $\operatorname{CogPack} \AA$ patients scoring significantly higher on delusional severity and positive symptoms, while patients in the MCT+ group had significantly more negative symptoms (Table 1).

Assessment data were available for approximately $86 \%$ of patients at $\mathrm{T} 1$ and $80 \%$ at $\mathrm{T} 2$ and did not significantly differ between the two groups. Attendance rates were significantly different between the two groups, which was due to higher early drop-out rates in the $\operatorname{CogPack}{ }^{\circledR}$ group. Patients who dropped out of treatment early (before the $4^{\text {th }}$ session) had significantly lower premorbid IQ ( $\mathrm{t}=1.95, \mathrm{p}=0.05)$, requested less draws to reach a decision in the Fish Task $(\mathrm{t}=3.57$, $\mathrm{p}=0.001)$, and had higher BCIS self-certainty scores $(\mathrm{t}=2.04, \mathrm{p}=0.04)$, as well as higher baseline disorganization $(t=2.01, p=0.05)$ and excitement scores $(t=2.66, p=0.009)$.

\subsection{Intervention effects}

Changes in outcome variables over time are presented in Table 2. Both ITT and PP analyses yielded similar results. There was a significant difference in favor of MCT+ with respect to the primary outcome variable, PSYRATS delusion score, at T1 [ITT: $p=0.03$; PP: $F(1,72)=5.89$, 
$\left.\mathrm{p}=0.02, \eta^{2} \mathrm{p}=0.08\right]$. The same was the case for PANSS item P1 at T1 [ITT: $p=0.04$; PP: $\left.F(1,72)=5.19, p=0.03, \eta^{2}=0.07\right]$.

Regarding reasoning, there were no differences in draws to conclusion change scores between the two groups $(\mathrm{p}>0.80)$. However, patients in the MCT+ group demonstrated a trend towards greater increase in their probability threshold to decision between baseline and T1 (ITT: $p=0.07$; PP: $\left.F(1,68)=3.38, p=0.07, \eta^{2}=0.05\right)$. Moreover, there was a significant difference in favor of $\mathrm{MCT}+$ regarding BCIS self-reflectiveness increase at T1 [ITT: $p=0.02 ; \mathrm{PP}: \mathrm{F}(1,72)=6.16, p=0.02$, $\left.\eta^{2} \mathrm{p}=0.08\right]$.

Regarding all other outcome variables, there were no significant differences between the two groups at T1, although there was a numerical advantage for MCT+ in most cases. The highest effect sizes, which bordered a statistical trend in the ITT analyses, were noted for WHOQOLBREF environment [ITT: $p=0.08 ; F(1,70)=2.60, p=0.11, \eta^{2}{ }_{p}=0.04$ ], and for self-esteem as assessed with the Rosenberg scale [ITT: $p=0.10 ; F(1,72)=2.50, p=0.12, \eta^{2}=0.03$ ].

At T2, there were no significant differences between the two groups in any of the psychopathology, reasoning or other variables (all $p>0.30$ ). In many cases, this was due to the fact that patients in the $\operatorname{CogPack}{ }^{\circledR}$ group, but not in the MCT+ group, showed further improvement between T1 and T2 (see Table 2).

\subsection{Additional analyses}

In order to assess whether concurrent group MCT affected results, we repeated all analyses using group MCT participation and its interaction with intervention group as additional predictors. The main effect of intervention group (MCT+ vs CogPack $®$ ) at T1 remained significant for PANSS P1 [ITT: $p=0.02$; PP: $F(1,68)=6.40, p=0.01, \eta^{2}=0.09$ ], BCIS selfreflectiveness score [ITT: $p=0.005$, PP: $F(1,68)=8.56, p=0.005, \eta^{2} p^{2} 0.11$ ], and for PSYRATS delusion score [ITT: $\mathrm{p}=0.04$; PP: $F(1,68)=7.18, \mathrm{p}=0.009, \eta^{2} \mathrm{p}=0.10$ ]. Regarding decision thresholds at T1, group MCT participation showed a trend-wise effect [ITT: $p=0.07 ; P P: F(1,68)=3.10$, $\left.p=0.08, \eta^{2}=0.05\right]$; the main effect of intervention group was no longer significant. At $T 2$, these additional analyses led to no differences compared to the original results.

We also repeated analyses including only patients who completed at least 4 sessions of either intervention. The cut-off of 4 sessions was selected to ensure that patients in the MCT+ group would have received at least one session dealing with cognitive biases. This change generally led to an increase of effect sizes in favor of MCT + at T1: PSYRATS delusions [ITT: $p=0.002$; PP: $F(1,59)=12.26, p=0.001, \eta^{2}{ }_{p}=0.17$ ]; P1 [ITT: $p=0.003 ; P P: F(1,59)=10.92, p=0.002, \eta^{2}{ }_{p}=0.16$ ]; BCIS self-reflectiveness [ITT: $p=0.002 ; \mathrm{PP}: \mathrm{F}(1,59)=13.1, \mathrm{p}=0.001, \eta^{2} \mathrm{p}=0.18$ ]. Moreover, significance was achieved at T1 for group differences in PANSS positive symptom improvement 
[ITT: $p=0.02 ;$ PP: $\left.\left.F(1,59)=6.00, p=0.02, \eta^{2}{ }^{2}=0.09\right)\right]$, PANSS total score improvement [ITT: $p=0.02$, PP: $\left.F(1,59)=6.69, p=0.01, \eta^{2}=0.10\right]$ and decision threshold increase [at a trend level for ITT: $\left.p=0.07 ; P P: F(1,57)=5.33, p=0.03, \eta^{2}=0.09\right]$. Results at $T 2$ did not change substantially.

\section{Discussion}

The present study assessed the efficacy of an individualized metacognitive intervention (MCT + ) compared to an active control, using a randomized controlled rater-blind design. MCT+ led to greater improvement regarding delusions and some aspects of cognitive bias in the short term (6-week follow-up). At the long-term follow-up 6 months later, there were no differences between the two intervention groups.

The beneficial effects of MCT + on delusions and (partly) cognitive biases are consistent with previous studies on group MCT in a purely group format (Moritz et al., 2014a) and accompanied by individual therapy sessions (Moritz et al., 2011). This effect was more pronounced in the subset of patients who attended a minimum of 4 sessions of either intervention (although this finding should be interpreted with caution, because it resulted from additional analyses that did not consider the original randomized patient sample). Importantly, the intervention was delivered by therapists who did not always have long experience; five of six therapists were still in psychotherapy training. It is possible that, due to its highly structured and manualized format, MCT+ might be suitable for low-threshold administration without extensive therapist training. This increased applicability might, in turn, improve dissemination in clinical practice, which is unfortunately still very low for evidence-based therapies such as CBT in patients with psychosis (Haddock et al., 2014).

The observed effects of MCT+ were only observable at the 6-week follow-up. The pattern of results suggests that, although $\mathrm{MCT}+$ led to improvement of delusions and reasoning quite early on (6 weeks), patients in the $\operatorname{CogPack} \AA$ group eventually attained the same improvement levels. This absence of longer-term effects is not consistent with previous studies on group MCT (Moritz et al., 2014a). It is probable that this inconsistency is due to selective floor effects in the MCT+ group: As shown in Tables 1 and 2, the randomization process resulted in differences between the two groups regarding baseline symptoms, which were lower in MCT+ patients -and, in fact, lower than in previous studies by our group (Moritz et al., 2013a; Moritz et al., 2011) and others (Favrod et al., 2014; So et al., 2015). This, in combination with the rapid improvement, led to very low symptom levels in these patients post-intervention, while the control group may have benefited from greater margins for change. Alternatively, it may be that further measures are needed to promote sustainability of MCT+ effects, especially in patients with psychosis who are known to have impaired memory capacity. The CBT concept of 'booster sessions' might be 
applicable here. Other, more low-threshold, possibilities include online or mobile-phone based exercises; our group is currently working on the development of respective applications.

Surprisingly, we did not observe a significant effect on the number of draws to decision in the Fish Task, inconsistent with our previous findings regarding group MCT (Moritz et al., 2013a). Table 1 indicates a possible reason for this negative finding: The number of draws to decision in the present patient sample was much higher at baseline compared to previous studies by our group and others (Moritz et al., 2013a; Moritz et al., 2011; So et al., 2015) on MCT. The Fish task is widely used in patient studies in our research center, and group MCT is an integral part of standard patient care in our department. Hence, most patients would have had at least some superficial contact with the concept of "hasty decisions", which may have biased findings. This is only a tentative explanation, since Garety et al. (2015) noted a significant effect of their own individualized metacognitive intervention on the number of draws to decision despite a similarly high baseline value as in the present study. In any event, MCT+ did have beneficial effects on other indices of cognitive bias (decision threshold and BCIS self-reflectiveness). However, these too were short-lived suggesting a possible need for 'reminders' in the forms discussed above. Interestingly, group differences between groups disappeared when concurrent participation in group MCT was considered in analyses, while a trend for the group intervention emerged. This may indicate a stronger effect for the group intervention, which focuses more explicitly on 'formal' aspects of cognitive biases rather than individual delusional content. However, this hypothesis needs to be assessed in future studies, as group MCT participation was not randomized.

Apart from a transient effect on WHOQOL-BREF environment factor at T1, we did not note any significant effects on quality of life and self-esteem, which may be due to the short follow-up period: in a previous trial by our group on group MCT (Moritz et al., 2014b), positive effects of MCT on quality of life and self-esteem were visible only at the 3-year follow-up.

A strength of the present study was that it used a randomized controlled rater-blind design while simulating conditions of standard care as well as possible through non-restrictive inclusion criteria, a wide range of patient baseline symptom severity and therapist experience, and flexibility regarding therapy content. However, there are also limitations that should be considered. (1) One limitation results from the significantly lower psychopathology scores of the MCT+ group at baseline. This difference between the two intervention groups can be attributed to chance, since our randomization plan was analogous to the one successfully implemented in a previous clinical trial by our group (Moritz et al., 2014b; Moritz et al., 2013a). Still, and although all analyses of clinical variables were adjusted for baseline symptom severity, it complicates interpretation of results: apart from issues associated with possible floor effects in the MCT+ group or larger regression to the mean in control group that may have confounded between- 
group differences, it is not possible to exclude subtle indirect effects of baseline psychopathology on outcomes, e.g. by affecting motivation for treatment, or the establishment of a therapeutic relationship. (2) There were significantly lower attendance rates in the CogPack $₫$ than in the MCT+ group. This is in itself a positive finding, as it reflects better acceptance rates of MCT+, confirming findings of previous studies on group MCT (Eichner and Berna, 2016). Moreover, attendance rates did not affect re-assessment rates, which were comparable in the two intervention groups, such that the validity of statistical analyses was not compromised. However, the two intervention groups cannot be considered to be similar regarding therapeutic effort. This is not a trivial matter, given the results of a recent meta-analysis on CBT for psychosis (Mehl et al., 2015) suggesting that effect sizes in favor of CBT diminish significantly when the intervention is not compared against treatment-as-usual, but rather against an active control intervention. Thus, this point should be considered when designing future efficacy studies for any psychotherapy approach. (3) A final limitation of the present study was that it did not include a group MCT arm. Although MCT group participation was considered in analyses, it will be an interesting aim for future studies to directly compare the two interventions, in order to assess whether the individualized format of MCT+ provides further advantages compared to the group training.

In summary, a short course of individualized metacognitive therapy $(\mathrm{MCT}+$ ) led to faster improvement in delusions and cognitive biases compared to a control intervention. The benefit associated with MCT+ disappeared in the long term, which may have been due to floor effects but may also indicate the need for regular reviews of therapeutic material after completion of the intervention.

\section{Acknowledgments}

This project was supported in part by a 2012 NARSAD Young Investigator Grant to the first author from the Brain \& Behavior Research Foundation. 


\section{Figure legends}

Figure 1: CONSORT flow diagram 


\section{Tables}

Table 1: Sample description and treatment characteristics

\begin{tabular}{|c|c|c|c|c|c|c|c|c|}
\hline & & MCT+ & & & $\overline{\text { CogPacl }}$ & & & \\
\hline & $n$ & mean & $S D$ & $n$ & mean & $S D$ & $t / \chi^{2}$ & $p$ \\
\hline Gender (m/f) & $21 / 25$ & & & $30 / 16$ & & & 3.56 & 0.09 \\
\hline Age (years) & & 36.91 & 12.5 & & 35.59 & 13.1 & 0.50 & 0.62 \\
\hline Years of education & & 11.65 & 1.7 & & 11.27 & 2.1 & 0.94 & 0.35 \\
\hline IQ & & 105.42 & 12.2 & & 100.91 & 11.5 & 1.78 & 0.08 \\
\hline \multicolumn{9}{|l|}{ Symptoms } \\
\hline PSYRATS delusions & & $5 \cdot 74$ & 5.9 & & 8.50 & $7 \cdot 3$ & 2.01 & 0.05 \\
\hline \multicolumn{9}{|l|}{ PANSS } \\
\hline P1 (delusions) & & 2.59 & 1.3 & & 3.24 & 1.7 & 2.05 & 0.04 \\
\hline total score & & 49.78 & 13.0 & & 49.35 & 12.8 & 0.16 & 0.87 \\
\hline positive & & $7 \cdot 37$ & 3.1 & & 9.33 & 4.4 & 2.46 & 0.02 \\
\hline negative & & 10.83 & 4.8 & & 8.30 & 2.9 & 3.06 & 0.003 \\
\hline disorganization & & $5 \cdot 30$ & 2.2 & & 5.76 & 2.5 & 0.92 & 0.36 \\
\hline excitement & & 4.74 & 1.3 & & 5.17 & 1.5 & 1.48 & 0.14 \\
\hline depression & & 6.85 & 3.1 & & 5.74 & 2.7 & 1.83 & 0.07 \\
\hline \multicolumn{9}{|l|}{ Reasoning style } \\
\hline Fish Task - draws to decision & & 4.02 & 2.8 & & 3.44 & 2.6 & 1.02 & 0.31 \\
\hline Fish Task - decision threshold & & 79.22 & 19.3 & & 78.47 & 22.5 & 0.17 & 0.87 \\
\hline BCIS self-certainty & & 13.84 & 2.8 & & 14.74 & 2.9 & 1.48 & 0.14 \\
\hline BCIS self-reflectiveness & & 23.89 & 4.1 & & 24.29 & 5.0 & 0.12 & 0.68 \\
\hline \multicolumn{9}{|l|}{ Quality of Life \& self-esteem } \\
\hline Rosenberg self-esteem scale & & 16.82 & 8.3 & & 19.82 & 8.2 & 1.72 & 0.89 \\
\hline \multicolumn{9}{|l|}{ WHOQOL-BREF } \\
\hline physical & & 57.48 & 20.8 & & 61.43 & 17.5 & 0.97 & 0.36 \\
\hline psychological & & 49.29 & 20.9 & & 56.53 & 19.3 & 1.69 & 0.10 \\
\hline relations & & 54.89 & 21.1 & & 57.27 & 21.8 & 0.52 & 0.60 \\
\hline environment & & 66.24 & 16.7 & & 62.97 & 19.1 & 0.86 & 0.39 \\
\hline \multicolumn{9}{|l|}{ Antipsychotic medication } \\
\hline $\mathrm{CPZ}$ dose at To & & 344.56 & 424.0 & & 305.49 & 393.5 & 0.85 & 0.40 \\
\hline mean CPZ dose To-T2 & & 343.39 & 310.2 & & 282.83 & 374.2 & 0.85 & 0.40 \\
\hline \multicolumn{9}{|l|}{ Attendance \& study adherence } \\
\hline number of sessions & & 8.28 & 3.5 & & 5.59 & 4.3 & 3.28 & 0.001 \\
\hline participation at $>=4$ sessions $(\mathrm{y} / \mathrm{n})$ & $41 / 5$ & & & $29 / 17$ & & & 8.60 & 0.006 \\
\hline data available at $\mathrm{T} 1(\mathrm{y} / \mathrm{n})^{*}$ & $42 / 4$ & & & $40 / 9$ & & & 2.24 & 0.23 \\
\hline data available at $\mathrm{T} 2(\mathrm{y} / \mathrm{n})^{* *}$ & $39 / 7$ & & & 35 / 11 & & & 1.11 & 0.43 \\
\hline
\end{tabular}


* for all variables, with the following exceptions: Fish Task based outcomes ( $n=40$ and $n=36$ cases for MCT + and $\operatorname{CogPack} 囚$, respectively); WHOQOL-BREF ( $\mathrm{n}=42$ and $n=35$ cases for $\mathrm{MCT}+$ and $\operatorname{CogPack} \AA$, respectively).

** for all variables, with the following exceptions: Fish Task based outcomes $(n=37$ and $n=35$ cases for MCT+ and $\operatorname{CogPack} 囚$, respectively); BCIS, WHOQOL-BREF and Rosenberg $(\mathrm{n}=40$ and $n=34$ cases for MCT + and $\operatorname{Cog} P a c k ®$, respectively). 
Table 2: Outcomes per intervention group and time point. Means and standard deviations (in brackets). Symbols indicate the significance levels for the comparison To-T1 and T1-T2 in each group separately. For between-group comparisons, please refer to the Results section.

\begin{tabular}{|c|c|c|c|c|c|c|}
\hline & \multicolumn{3}{|c|}{ MCT+ } & \multicolumn{3}{|c|}{ CogPack } \\
\hline & To & $\begin{array}{c}\text { T1 } \\
\text { (within-subject } \\
\text { change from To) }\end{array}$ & $\begin{array}{c}\text { T2 } \\
\text { (within-subject } \\
\text { change from T1) }\end{array}$ & To & $\begin{array}{c}\text { T1 } \\
\text { (within-subject } \\
\text { change from To) }\end{array}$ & $\begin{array}{c}\text { T2 } \\
\text { (within-subject } \\
\text { change from } T_{1} \text { ) }\end{array}$ \\
\hline Symptoms & & & & & & \\
\hline PSYRATS delusions & $5.74 \quad(5.9)$ & $3.48^{* *}(5.1)^{* *}$ & $5.05(6.6)$ & $8.50 \quad(7.3)$ & $7.43(7.1)^{\S}$ & $5.60(7.0)^{*}$ \\
\hline \multicolumn{7}{|l|}{ PANSS } \\
\hline P1 (delusions) & $2.59(1.3)$ & $1.88(1.0)^{* * *}$ & $2.10 \quad(1.4)$ & $3.24(1.7)$ & $2.68(1.5)^{* *}$ & $2.34(1.6)^{*}$ \\
\hline total score & 49.78 (13.0) & $43.31(9.2)^{* * *}$ & $43.85 \quad(12.8)$ & $49.35 \quad(12.8)$ & $45.43(11.0)^{* *}$ & $45.00 \quad(12.1)$ \\
\hline positive & $7.37 \quad(3.1)$ & $6.00(2.6)^{* * *}$ & $6.23(3.1)$ & $9.33(4.4)$ & $7.70(3.5)^{* *}$ & $7.66 \quad(3.4)$ \\
\hline negative & $10.83(4.8)$ & $9.64(4.7)^{*}$ & $9.35 \quad(3.9)$ & $8.30 \quad(2.9)$ & $8.24(3.3)^{*}$ & $8.37 \quad(3.0)$ \\
\hline disorganization & $5.30 \quad(2.2)$ & $4.50(1.7)^{* *}$ & $4.58 \quad(2.0)$ & $5.76 \quad(2.5)$ & 4.84 (1.9) & $4.91 \quad(2.6)$ \\
\hline excitement & $4.74 \quad(1.3)$ & $4.45(0.9)$ & 4.38 (o.8) & $5.17(1.5)$ & $4.92(1.6)$ & $4.94 \quad(1.7)$ \\
\hline depression & $6.85(3.1)$ & $5.76(2.6)^{*}$ & $5.85 \quad(3.3)$ & $5.74 \quad(2.7)$ & $5.49 \quad(2.5)$ & $5.23(3.0)$ \\
\hline \multicolumn{7}{|l|}{ Reasoning Style } \\
\hline Fish Task - draws to decision & $4.02(2.8)$ & $4.10 \quad(2.3)$ & $4.19 \quad(2.4)$ & $3.44(2.6)$ & $4.22(2.9)$ & $4.49 \quad(2.9)$ \\
\hline Fish Task - decision threshold & $79.22(19.3)$ & $83.69(18.3)^{\S}$ & $82.81 \quad(18.8)$ & $78.47(22.5)$ & $74.08 \quad(28.0)$ & $84.31 \quad(19.6)$ \\
\hline BCIS self-certainty & $13.84(2.8)$ & $13.98(2.5)$ & $14.14(2.8)$ & $14.74(2.9)$ & $14.08 \quad(2.8)$ & $13.96(2.4)$ \\
\hline BCIS self-reflectiveness & $23.89 \quad(4.1)$ & $24.76(4.4)^{*}$ & $23.28(4.6)^{*}$ & $24.29(5.0)$ & $23.04(4.5)$ & $22.80 \quad(4.9)$ \\
\hline \multicolumn{7}{|l|}{ Quality of Life \& self-esteem } \\
\hline Rosenberg self-esteem scale & $16.82(8.3)$ & $20.17(8.2)^{* * *}$ & $20.65(7.7)$ & $19.82(8.2)$ & $20.59(7.4)$ & $21.79(7.9)^{*}$ \\
\hline \multicolumn{7}{|l|}{ WHOQOL-BREF } \\
\hline physical & $57.48 \quad(20.8)$ & $63.48(18.5)^{*}$ & $66.52(18.1)$ & $61.43(17.5)$ & $64.59(18.3)$ & $64.18 \quad(19.3)$ \\
\hline psychological & $49.29(20.9)$ & $56.57(20.3)^{*}$ & $60.52(19.0)^{\S}$ & $56.53(19.3)$ & $61.31 \quad(17.8)$ & $61.11(17.4)$ \\
\hline relations & $54.89(21.1)$ & $59.03(22.3)$ & $63.13(19.2)$ & $57.27(21.8)$ & $57.14 \quad(20.4)$ & $61.74 \quad(17.3)$ \\
\hline environment & $66.24(16.7)$ & $71.00(13.3)^{*}$ & $72.47 \quad(15.9)$ & $62.97(19.1)$ & $65.92(15.5)$ & $67.55 \quad(13.9)$ \\
\hline
\end{tabular}




\section{References}

Balzan, R.P., Delfabbro, P.H., Galletly, C.A., Woodward, T.S., 2014. Metacognitive training for patients with schizophrenia: preliminary evidence for a targeted, single-module programme. Aust N Z J Psychiatry 48(12), 1126-1136.

Balzan, R.P., Galletly, C., 2015. Metacognitive therapy (MCT+) in patients with psychosis not receiving antipsychotic medication: A case study. Front Psychol 6, 967.

Beck, A.T., Baruch, E., Balter, J.M., Steer, R.A., Warman, D.M., 2004. A new instrument for measuring insight: the Beck Cognitive Insight Scale. Schizophr Res 68(2-3), 319-329.

Beck, A.T., Warman, D.M., 2004. Cognitive insight: Theory and assessment, in: Amador, X.F., David, A.S. (Eds.), Insight and Psychosis: Awareness of Illness in Schizophrenia and Related Disorders, 2nd ed. Oxford University Press, New York, NY, pp. 79-87.

Drake, R., Haddock, G., Tarrier, N., Bentall, R., Lewis, S., 2007. The Psychotic Symptom Rating Scales (PSYRATS): their usefulness and properties in first episode psychosis. Schizophr Res 89(1-3), 119-122.

Eichner, C., Berna, F., 2016. Acceptance and Efficacy of Metacognitive Training (MCT) on Positive Symptoms and Delusions in Patients With Schizophrenia: A Meta-analysis Taking Into Account Important Moderators. Schizophr Bull.

Erdfelder, E., Faul, F., Buchner, A., 1996. GPOWER: A general power analysis program. Behavior Research Methods, Instruments, \& Computers 28, 1-11.

Favrod, J., Rexhaj, S., Bardy, S., Ferrari, P., Hayoz, C., Moritz, S., Conus, P., Bonsack, C., 2014. Sustained antipsychotic effect of metacognitive training in psychosis: a randomized-controlled study. Eur Psychiatry 29(5), 275-281.

Freeman, D., Dunn, G., Startup, H., Pugh, K., Cordwell, J., Mander, H., Cernis, E., Wingham, G., Shirvell, K., Kingdon, D., 2015. Effects of cognitive behaviour therapy for worry on persecutory delusions in patients with psychosis (WIT): a parallel, single-blind, randomised controlled trial with a mediation analysis. Lancet Psychiatry 2(4), 305-313.

Garety, P., Waller, H., Emsley, R., Jolley, S., Kuipers, E., Bebbington, P., Dunn, G., Fowler, D., Hardy, A., Freeman, D., 2015. Cognitive mechanisms of change in delusions: an experimental investigation targeting reasoning to effect change in paranoia. Schizophr Bull 41(2), 400-410.

Garety, P.A., Freeman, D., 2013. The past and future of delusions research: from the inexplicable to the treatable. Br J Psychiatry 203(5), 327-333.

Haddock, G., Eisner, E., Boone, C., Davies, G., Coogan, C., Barrowclough, C., 2014. An investigation of the implementation of NICE-recommended CBT interventions for people with schizophrenia. J Ment Health 23(4), 162-165.

Haddock, G., McCarron, J., Tarrier, N., Faragher, E.B., 1999. Scales to measure dimensions of hallucinations and delusions: the psychotic symptom rating scales (PSYRATS). Psychol Med $29(4), 879-889$.

Hutton, P., Taylor, P.J., 2014. Cognitive behavioural therapy for psychosis prevention: a systematic review and meta-analysis. Psychol Med 44(3), 449-468.

Jaaskelainen, E., Juola, P., Hirvonen, N., McGrath, J.J., Saha, S., Isohanni, M., Veijola, J., Miettunen, J., 2013. A systematic review and meta-analysis of recovery in schizophrenia. Schizophr Bull 39(6), 1296-1306.

Jaspers, K., 1913. Allgemeine Psychopathologie. Springer, Berlin, Germany. 
Jauhar, S., McKenna, P.J., Radua, J., Fung, E., Salvador, R., Laws, K.R., 2014. Cognitive-behavioural therapy for the symptoms of schizophrenia: systematic review and meta-analysis with examination of potential bias. Br J Psychiatry 204(1), 20-29.

Kay, S.R., Fiszbein, A., Opler, L.A., 1987. The positive and negative syndrome scale (PANSS) for schizophrenia. Schizophr Bull 13(2), 261-276.

Kinnear, P.R., Gray, C.D., 2009. SPSS 16 - Made Simple. Taylor \& Francis, London.

Leucht, S., Arbter, D., Engel, R.R., Kissling, W., Davis, J.M., 2009. How effective are secondgeneration antipsychotic drugs? A meta-analysis of placebo-controlled trials. Mol Psychiatry 14(4), 429-447.

Lieberman, J.A., Stroup, T.S., McEvoy, J.P., Swartz, M.S., Rosenheck, R.A., Perkins, D.O., Keefe, R.S., Davis, S.M., Davis, C.E., Lebowitz, B.D., Severe, J., Hsiao, J.K., Clinical Antipsychotic Trials of Intervention Effectiveness, I., 2005. Effectiveness of antipsychotic drugs in patients with chronic schizophrenia. N Engl J Med 353(12), 1209-1223.

Lynch, D., Laws, K.R., McKenna, P.J., 2010. Cognitive behavioural therapy for major psychiatric disorder: does it really work? A meta-analytical review of well-controlled trials. Psychol Med 40(1), 9-24.

Mander, H., Kingdon, D., 2015. The evolution of cognitive-behavioral therapy for psychosis. Psychol Res Behav Manag 8, 63-69.

Marker, K., 2003. COGPACK Manual Version 5.9. . Marker Software, Ladenburg.

McKenna, P., Kingdon, D., 2014. Has cognitive behavioural therapy for psychosis been oversold? BMJ 348, g2295.

Mehl, S., Werner, D., Lincoln, T.M., 2015. Does Cognitive Behavior Therapy for psychosis (CBTp) show a sustainable effect on delusions? A meta-analysis. Front Psychol 6, 1450.

Moritz, S., Andreou, C., Schneider, B.C., Wittekind, C.E., Menon, M., Balzan, R.P., Woodward, T.S., 2014a. Sowing the seeds of doubt: a narrative review on metacognitive training in schizophrenia. Clin Psychol Rev 34(4), 358-366.

Moritz, S., Van Quaquebeke, N., Lincoln, T.M., 2012a. Jumping to conclusions is associated with paranoia but not general suspiciousness: a comparison of two versions of the probabilistic reasoning paradigm. Schizophr Res Treatment 2012, 384039.

Moritz, S., Veckenstedt, R., Andreou, C., Bohn, F., Hottenrott, B., Leighton, L., Kother, U., Woodward, T.S., Treszl, A., Menon, M., Schneider, B.C., Pfueller, U., Roesch-Ely, D., 2014b. Sustained and "sleeper" effects of group metacognitive training for schizophrenia: a randomized clinical trial. JAMA Psychiatry 71(10), 1103-1111.

Moritz, S., Veckenstedt, R., Bohn, F., Hottenrott, B., Scheu, F., Randjbar, S., Aghotor, J., Kother, U., Woodward, T.S., Treszl, A., Andreou, C., Pfueller, U., Roesch-Ely, D., 2013a. Complementary group Metacognitive Training (MCT) reduces delusional ideation in schizophrenia. Schizophr Res 151(1-3), 61-69.

Moritz, S., Veckenstedt, R., Bohn, F., Köther, U., Woodward, T.S., 2013b. Metacognitive training in schizophrenia. Theoretical rationale and administration, in: Roberts, D.L., Penn, D.L. (Eds.), Social cognition in schizophrenia. From evidence to treatment. Oxford University Press, New York, pp. 358-383.

Moritz, S., Veckenstedt, R., Randjbar, S., Vitzthum, F., 2012b. Individualisierte Metakognitive Therapie für Menschen mit Psychose (MKT+). Springer, Heidelberg, Germany.

Moritz, S., Veckenstedt, R., Randjbar, S., Vitzthum, F., Woodward, T.S., 2011. Antipsychotic treatment beyond antipsychotics: metacognitive intervention for schizophrenia patients improves delusional symptoms. Psychol Med 41(9), 1823-1832. 
Moritz, S., Woodward, T.S., Burlon, M., 2005. Metacognitive skill training for patients with schizophrenia (MCT). Manual. VanHam Campus Verlag, Hamburg

Mueser, K.T., Deavers, F., Penn, D.L., Cassisi, J.E., 2013. Psychosocial treatments for schizophrenia. Annu Rev Clin Psychol 9, 465-497.

Murphy, B., Herrman, H., Hawthorne, G., Pinzone, T., Evert, H., 2000. Australian WHOQoL instruments: User's manual and interpretation guide. . Australian WHOQoL Field Study Centre, Melbourne, Australia.

Peralta, V., Cuesta, M.J., 1994. Psychometric properties of the positive and negative syndrome scale (PANSS) in schizophrenia. Psychiatry Res 53(1), 31-40.

Riggs, S.E., Grant, P.M., Perivoliotis, D., Beck, A.T., 2012. Assessment of cognitive insight: a qualitative review. Schizophr Bull 38(2), 338-350.

Ross, K., Freeman, D., Dunn, G., Garety, P., 2011. A randomized experimental investigation of reasoning training for people with delusions. Schizophr Bull 37(2), 324-333.

Schmidt, K.H., Metzler, P., 1992. Wortschatztest. Hogrefe, Göttingen.

Sheehan, D.V., Lecrubier, Y., Sheehan, K.H., Amorim, P., Janavs, J., Weiller, E., Hergueta, T., Baker, R., Dunbar, G.C., 1998. The Mini-International Neuropsychiatric Interview (M.I.N.I.): the development and validation of a structured diagnostic psychiatric interview for DSM-IV and ICD10. J Clin Psychiatry 59 Suppl 20, 22-33; quiz 34-57.

So, S.H., Chan, A.P., Chong, C.S., Wong, M.H., Lo, W.T., Chung, D.W., Chan, S.S., 2015. Metacognitive training for delusions (MCTd): effectiveness on data-gathering and belief flexibility in a Chinese sample. Front Psychol 6, 730.

Turner, D.T., van der Gaag, M., Karyotaki, E., Cuijpers, P., 2014. Psychological interventions for psychosis: a meta-analysis of comparative outcome studies. Am J Psychiatry 171(5), 523-538.

van Oosterhout, B., Krabbendam, L., de Boer, K., Ferwerda, J., van der Helm, M., Stant, A.D., van der Gaag, M., 2014. Metacognitive group training for schizophrenia spectrum patients with delusions: a randomized controlled trial. Psychol Med 44(14), 3025-3035.

van Oosterhout, B., Smit, F., Krabbendam, L., Castelein, S., Staring, A.B., van der Gaag, M., 2016. Metacognitive training for schizophrenia spectrum patients: a meta-analysis on outcome studies. Psychol Med 46(1), 47-57.

von Collani, G., Herzberg, P.Y., 2003. Eine revidierte Fassung der deutschsprachigen Skala zum Selbstwertgefühl von Rosenberg. [A revised version of the German adaption of Rosenberg's SelfEsteem Scale). Zeitschrift für Differ und Diagnostische Psychol 2003(24), 3-7.

Waller, H., Freeman, D., Jolley, S., Dunn, G., Garety, P., 2011. Targeting reasoning biases in delusions: a pilot study of the Maudsley Review Training Programme for individuals with persistent, high conviction delusions. J Behav Ther Exp Psychiatry 42(3), 414-421.

Wallwork, R.S., Fortgang, R., Hashimoto, R., Weinberger, D.R., Dickinson, D., 2012. Searching for a consensus five-factor model of the Positive and Negative Syndrome Scale for schizophrenia. Schizophr Res 137(1-3), 246-250.

Wykes, T., Steel, C., Everitt, B., Tarrier, N., 2008. Cognitive behavior therapy for schizophrenia: effect sizes, clinical models, and methodological rigor. Schizophr Bull 34(3), 523-537. 


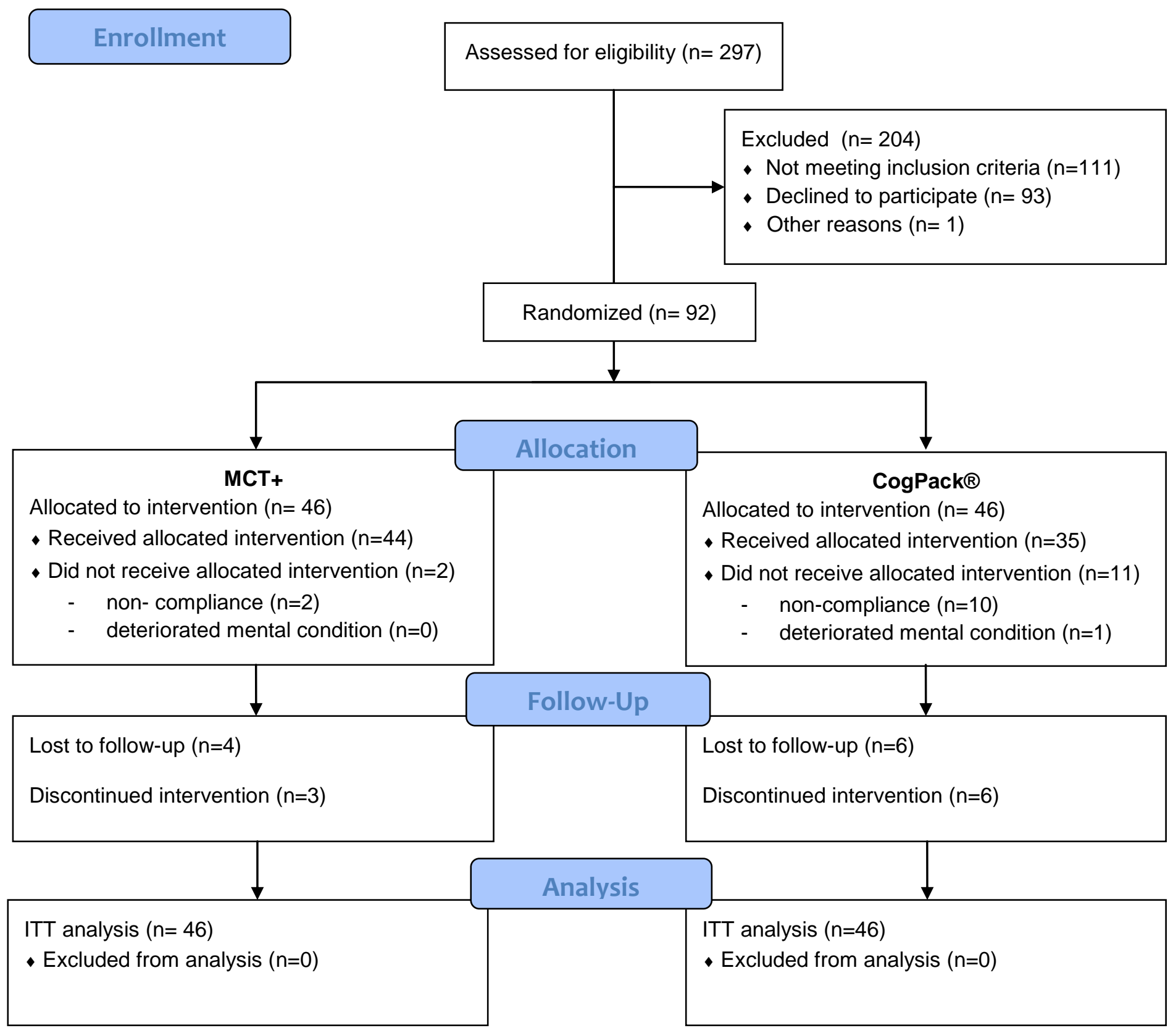

